\title{
Differentiation analyses of adult suspension mononucleated peripheral blood cells of Mus musculus
}

\author{
Shahrul Hisham Zainal Ariffin ${ }^{1 *}$, Intan Zarina Zainol Abidin ${ }^{1 \dagger}$, Muhammad Dain Yazid ${ }^{1}$, \\ Rohaya Megat Abdul Wahab²
}

\begin{abstract}
Background: The purpose of this study is to determine whether isolated suspension mouse peripheral mononucleated blood cells have the potential to differentiate into two distinct types of cells, i.e., osteoblasts and osteoclasts.

Results: Differentiation into osteoblast cells was concomitant with the activation of the Opn gene, increment of alkaline phosphatase (ALP) activity and the existence of bone nodules, whereas osteoclast cells activated the Catk gene, increment of tartrate resistant acid phosphatase (TRAP) activity and showed resorption activities via resorption pits. Morphology analyses showed the morphology of osteoblast and osteoclast cells after von Kossa and May-Grunwald-Giemsa staining respectively.
\end{abstract}

Conclusions: In conclusion, suspension mononucleated cells have the potentiality to differentiate into mature osteoblasts and osteoclasts, and hence can be categorized as multipotent stem cells.

\section{Background}

Stem cells, or mother cells, are cells with the ability to divide for indefinite periods in time and to give rise to specific cells. The primitive stage of stem cells can be divided into three types; i.e., totipotent, pluripotent and multipotent cells $[1,2]$. Totipotent cells are the most primitive cells, followed by pluripotent cells. The multipotent cell type is the most differentiated type of stem cells [3]. Osteoblasts and osteoclasts are cells that are responsible for bone formation and resorption respectively [4]. During bone formation, osteoblasts deposit the organic and inorganic matrix, whilst osteoclasts remove bone matrix [5]. Osteoblast and osteoclast cells are originated from different lineage, i.e., osteoblasts arise from mesenchymal stem cells and osteoclasts originate from haematopoietic stem cells [6].

The objective of this study is to determine the potentiality of isolated suspension mononucleated cells to differentiate into osteoblast and osteoclast cells. Osteoblasts

\footnotetext{
* Correspondence: shahroy8@gmail.com

+ Contributed equally

'School of Biosciences and Biotechnology, Faculty of Science and

Technology, Universiti Kebangsaan Malaysia, 43600 Bangi, Selangor, Malaysia

Full list of author information is available at the end of the article
}

were originated from different lineage (mesenchymal stem cells) but osteoclasts were originated from haematopoietic stem cells. This indicates that the isolated mononucleated cells capable to differentiate into more than one type of cells. Our results show that suspension mononucleated cells isolated from peripheral mouse blood have the potential to develop into more than one type of mature cell. The results also demonstrate the plasticity of adult stem cells isolated from peripheral blood. These cells are capable of fully differentiating into osteoblast and osteoclast cells. The presences of osteoblast and osteoclast cells were determined using molecular biology, cells activity and morphology analyses.

\section{Results and Discussion}

\section{Proliferation of Mononucleated Cells}

The heterogenic populations were characterised using cell culture selection. After 15 days cultured in selection medium, majority of differentiated and precursor cells died due to short lifespans, e.g., granulocytes (30-40 minutes in the peripheral blood with a total lifespan of 7-13 days that varied under certain pathological conditions) [7], monocytes (5-7 days) [8] and platelets (3-5 days) [9]. 
As a result, only primitive mononucleated cells (stem cells) survive after 15 days cultured in selection medium.

Besides differentiation potential, self-renewal is the main property of stem cells. The suspension mononucleated cells population doubling times are 3.1 days [10]. Passages were done every doubling time i.e., 3-4 days. Within 15 days, 4-5 passages were used for each isolates which produce a total cell number of approximately 3-5 $\times 10^{6}$ cells $/ \mathrm{mL}$ for every isolates which start from approximately $1 \times 10^{5}$ cells $/ \mathrm{mL}$. As conclusion, within 15 days the suspension mononucleated cells were shown to proliferate in in vitro conditions.

\section{Activation of Molecular Markers in Differentiated Mononucleated Cells}

Previous studies have demonstrated that there are several specific gene markers for osteoblast cells, such as osteopontin, alkaline phosphatase, osteocalcin and collagen type I (Col1) [11,12]. In this study, molecular analysis was performed to determine in vitro differentiation of peripheral blood mononucleated cells into mature osteoblast cells after induction by differentiation factors, i.e., ascorbic acid and $\beta$-glycerophosphate, for 14 days.

The activation of the Opn gene shows that mononucleated cells have differentiated into osteoblast cells. RTPCR analysis showed different levels of Opn gene expression in mononucleated cells cultured in proliferation medium compared to osteoblast differentiation medium (Figure 1B). Both media produced the expected products; i.e., the Opn gene with size $\sim 234$ bp when amplified with RT-PCR. However, the expression of the Opn gene for mononucleated cells cultured in osteoblast differentiation medium were much higher as compared to mononucleated cells cultured in proliferation medium (Figure 1B). The increase in Opn gene transcription showed that peripheral blood mononucleated cells differentiate into mature osteoblast cells. Opn gene can produce low grade expression in variety of cells, such as fibroblast, differentiated osteoblast and osteoclast cells and bone marrow derived cells [13]. Low level expression of Opn gene in this study might be due to the existence a few of all these differentiated cells types and also primitive cells that can survive after 15 days of medium selection. However, the increment of expression level after been induced in specialized osteoblast medium was contributed by the primitive cell (stem cells) that have been differentiated into osteoblast cells.

RT-PCR analysis was done on day 14 based on the fact that the mononucleated cell line (MC3T3-E1) is fully differentiated into osteoblast cells after 14 days in differentiation medium [14]. Similarly, based on studies in rat calvarial osteoblast-like (ROB) cell, the Opn gene was also expressed during 13 to 15 days of differentiation [15]. Furthermore, the study showed that ROB cells

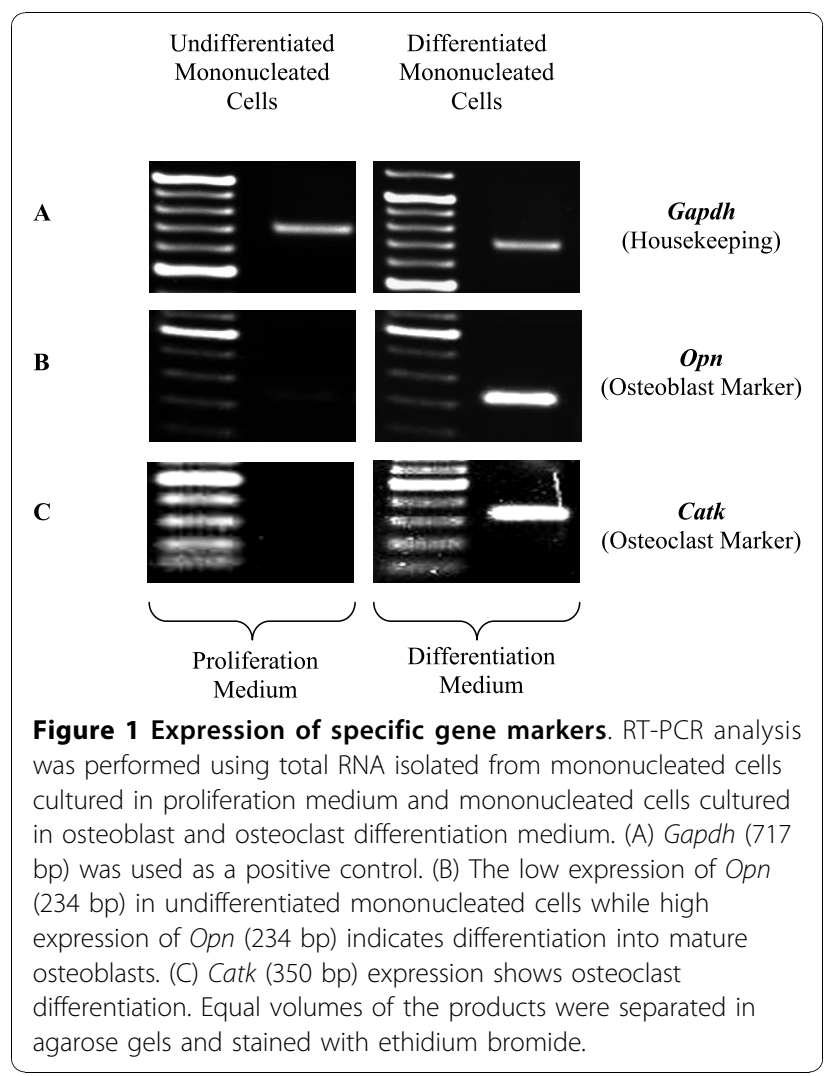

differentiate morphologically into mature osteoblast cells after 13 days of differentiation. Therefore, this study demonstrates that mononucleated cells cultured in osteoblast differentiation medium express the $\mathrm{Opn}$ gene when differentiated into osteoblast cells. In addition, alkaline phosphatase (ALP) assay also showed the increment of ALP activity after 10 and 14 days of mononucleated cells cultured in osteoblast differentiation medium compared to cells cultured in control medium (proliferation medium) (Figure 2). MC3T3-E1 cell line (pre-osteoblast) that cultured in differentiation medium (presence of ascorbic acid and $\beta$-glycerophosphate) exhibits high ALP activity after 10 and 14 days in the medium $[14,16]$. This is an indication that ALP is a marker for the bone formation. The expression of Opn gene and increment of ALP activity showed that mononucleated cells are differentiated into osteoblast cells.

In this study, the housekeeping gene Gapdh was used as a positive control for mononucleated cells in both types of medium, i.e., proliferation and differentiation medium. Figure 1A shows that RT-PCR amplification produces a Gapdh band ( $717 \mathrm{bp})$ from mononucleated cells in both types of media. The activation of the Gapdh gene proves that cells perform basic metabolic processes required for cell survival. An inherent assumption in the use of housekeeping genes is that expression of the genes 


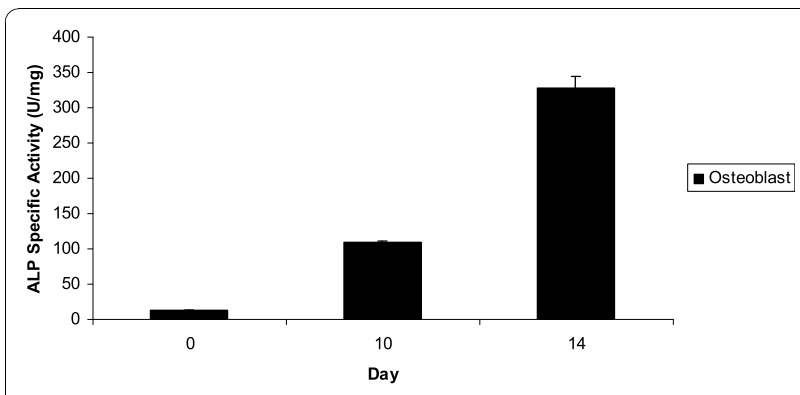

Figure 2 ALP specific activity for mononucleated cells cultured in osteoblast differentiation medium. ALP specific activity showed an increment after 14 days mononucleated cells cultured in osteoblast differentiation medium.

remains constant in the cells or in the tissues under investigation [17].

Differentiated osteoclast cells express specific markers, such as tartrate resistant acid phosphatase and cathepsin $\mathrm{K}[18,19]$. Therefore, the Catk gene will only be activated in osteoclast cells. Molecular analysis was performed to determine whether the isolated mononucleated cells had indeed differentiated into mature osteoclast cells. The activation of the Catk gene has been observed in mononucleated cells induced in vitro for osteoclast differentiation over a period of 10 days. RT-PCR analysis was done on day 10 since mononucleated cells are believed to differentiate into mature osteoclasts at this time point, thus suitable for molecular analysis [20-23].

The experimental results are shown in Figure $1 \mathrm{C}$. The RT-PCR analysis of the transcripts from mononucleated cells cultured in osteoclast differentiation medium showed the activation of the Catk gene. The RT-PCR product showed amplification of a band with the expected size for the Catk gene, i.e., 350 bp (Figure 1C). However, no band was found from mononucleated cells cultured in proliferation medium, which served as the negative control experiment (Figure 1C). Therefore, activation of the Catk gene does not occur during cells proliferation. This study showed that mononucleated cells cultured in osteoclast differentiation medium for 10 days activate the Catk gene, similar to mature osteoclast cells. In addition, the increment of tartrate resistant acid phosphatase (TRAP) activity in mononucleated cells cultured in osteoclast differentiation medium after 10 days compared to cells cultured in control medium (proliferation medium) also indicates that the cells are differentiated into osteoclast cells (Figure 3).

\section{Osteoblastic and Osteoclastic Cell Activities}

Cell activities determination using osteologic discs showed that discs cultured in osteoblast differentiation medium were positive for von Kossa staining, an indication of the

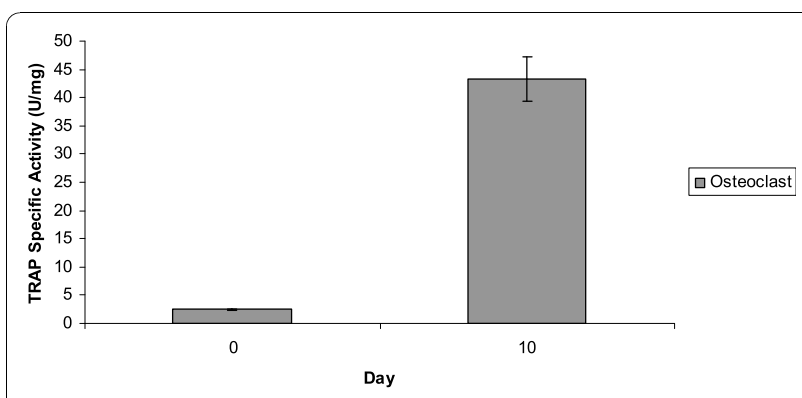

Figure 3 TRAP specific activity for mononucleated cells cultured in osteoclast differentiation medium. TRAP specific activity showed an increment after 10 days mononucleated cells cultured in osteoclast differentiation medium

existence of calcium nodules (Figure 4). Recent study by Sindrey et al. (1999) showed that generated calcium mineral by osteoblast cells is stained by von Kossa as black or yellow to brown based on the amount of secreted calcium matrices, whereas negative control disc is stained as light brown or gray [24]. The control disc cultured in proliferation medium did not show the formation of calcium nodules on the disc surface (Figure 4A). During differentiation, osteoblasts express the ALP enzyme, which utilizes phosphate from the medium deposits it on the disc surface, which then accumulates to become calcium nodules stained by von Kossa [12,25]. In our study, we compared our discs to the background colour (control disc; cell cultured without osteoblast differentiation factors), where the increment of intensity for the background during cell development has been observed. The background colour and quantity of bone nodules increase starting from day 5 until 14 (Figure 4B-E). Pre-osteoblasts produce high activities after culturing in differentiation medium consisting of ascorbic acid and $\beta$-glycerophosphate for 10 and 14 days $[14,16]$.

During our osteoclast differentiation assay, the formation of clear resorption pits on the disc surface due to the resorption of the calcium phosphate layer by osteoclast cells was observed (Figure 5). The resorption pits on the disc surface increased from day 5 until 10 (Figure 5B-D). Other studies also showed that osteoclasts are differentiated in culture by day 10 [20-22,26]. Studies by Valverde et al. showed that osteoclastic activity can be determined using bone resorption pits analysis that was performed on calcium phosphate disc (BD BioCoat ${ }^{\mathrm{TM}}$ Osteologic ${ }^{\mathrm{TM}}$ Disc) $[27,28]$. Therefore, our study involving similar disc was performed to determine the existence of in vitro osteoclastic activity. In addition, there were no resorption pits found on the disc surface for the control disc, indicating the absence of osteoclast activity in cells cultured in proliferation medium (Figure 5A). 


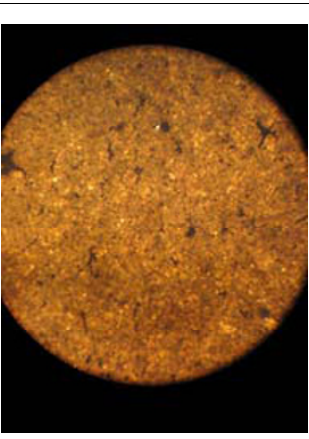

A) Control

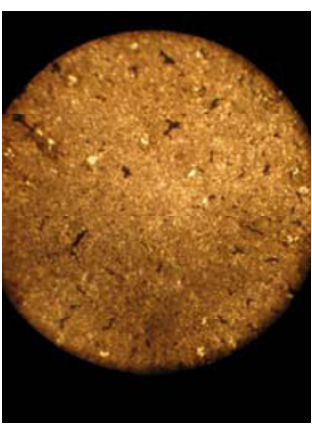

B) Day 5

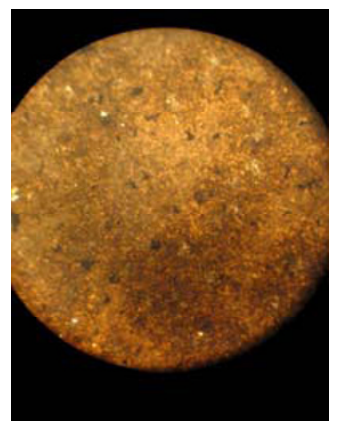

C) Day 7

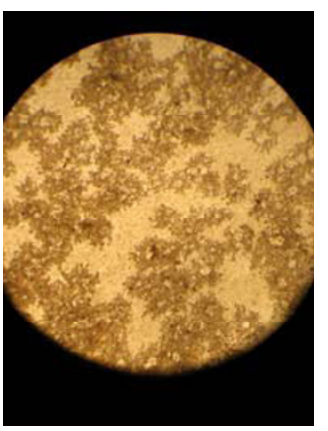

D) Day 10

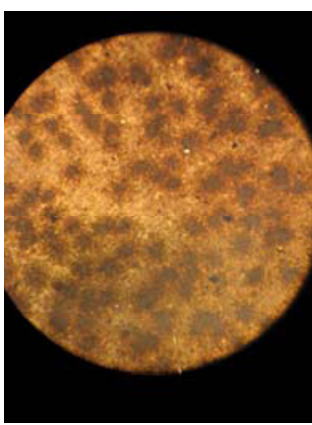

E) Day 14

Figure 4 Osteoblastic activity on osteologic disc in osteoblast differentiation medium. The discs' background colour became darker each day after von Kossa staining; an indication of the development of bone nodule (B-E) compared to control (A).

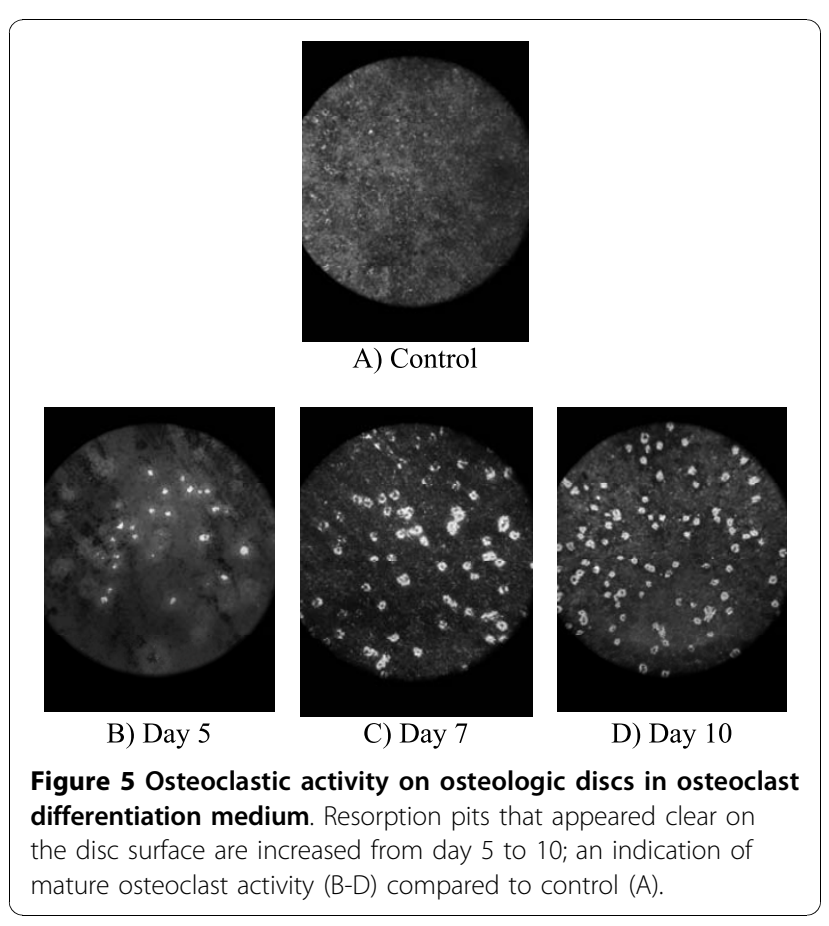

\section{Morphology of Differentiated Osteoblast and Osteoclast Cells}

The newly isolated mouse peripheral blood mononucleated cells stained by von Kossa (Figure 6A) and MayGrunwald-Giemsa (Figure 6B) were used as controls in this study. These cells were cultured in proliferation medium without any supplementation of differentiation factors. Morphologically, each cell has a large single nucleus that occupies much of the cytoplasmic space (Figures 6A and 6B). The black arrows show the morphology of mononucleated cells without any minerals and calcium deposited when cultured in the proliferation medium.

Figures $6 \mathrm{C}$ and $6 \mathrm{D}$ show mononucleated cells have been differentiated into osteoblasts and osteoclasts respectively. After von Kossa staining in mononucleated cells cultured in osteoblast differentiation medium for 14 days, mineral nodules were found deposited in these cytoplasmic cells (Figure 6C). According to Alhadlaq and Mao (2003), von Kossa staining can reveal mineral nodules in chondrogenic and osteogenic cells differentiated from mesenchymal stem cells [29]. Other studies also used von Kossa staining to observe the effect of stimulants on osteoblastic cells by quantifying the formation of mineralization nodules in osteoblasts cultures [30]. Therefore, isolated mononucleated cells cultured 


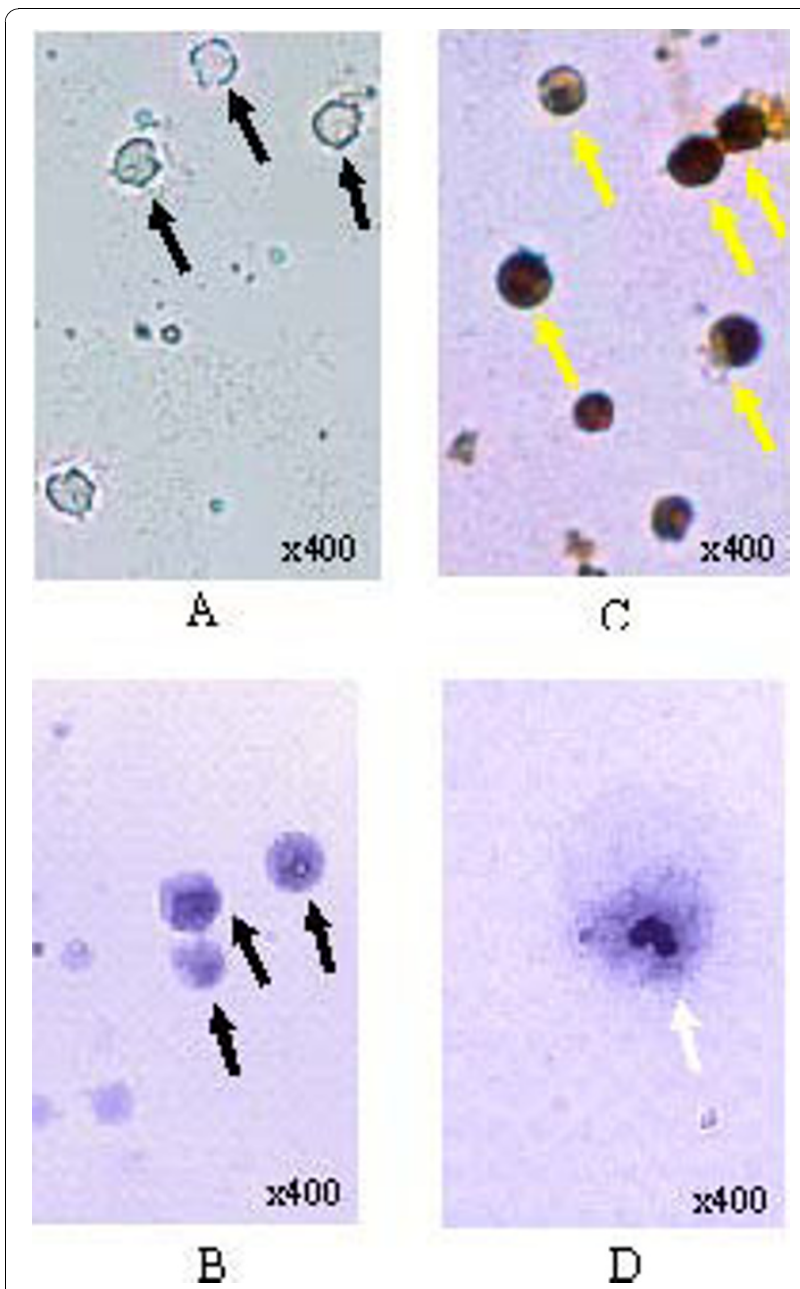

Figure 6 Morphology of mouse mononucleated cells. (A and B) Mononucleated cells cultured in proliferation medium stained by von Kossa and May-Grunwald-Giemsa, respectively. (C)

Mononucleated cells after 14 days cultured in osteoblast differentiation medium stained by von Kossa. (D) Mononucleated cells after 10 days cultured in osteoclast differentiation medium stained by May-Grunwald-Giemsa. Black arrows show undifferentiated mononucleated cells. Yellow arrows show the black granules in osteoblast cells. White arrow shows multiple nuclei and gigantic osteoclast cells.

for 14 days in osteoblast differentiation medium can differentiate into mature osteoblast cells.

Mature osteoclast cells are large multinucleated cells with 6-12 nuclei. Another morphological feature of osteoclasts is the presence of ruffled border and sealing zone $[31,32]$. May-Grunwald-Giemsa staining showed that these bone-resorbing cells contain many nuclei in its homogenous cytoplasm. May-Grunwald with conjuction of Giemsa is a classic haematology staining procedures that was used to stain peripheral blood and bone marrow specimen. This type of staining can differentiate between nucleus and cytoplasm, which nuclei and cytoplasm as in blue and pink-rose colour, respectively. Osteoclast distinct morphological feature is the presence of multinucleus cells which therefore can be detected using May-GrunwaldGiemsa [33]. After May-Grunwald-Giemsa staining of induced mononucleated cells, the nuclei are purple in colour, while the cytoplasm is lighter than the nucleus (Figure 6D). In this study, mononucleated cells cultured in osteoclast differentiation medium for 10 days showed the morphology of osteoclast cells. The large multinucleated osteoclast cells possessed four nuclei stained purple in colour (Figure 6D). Therefore, mononucleated cells have been differentiated into mature osteoclast cells.

During differentiation, not all cells are differentiated into osteoblast and osteoclast cells. After 14 days of osteoblast differentiation, there were only about $98 \%$ of mononucleated cells are differentiated into osteoblast (Figure 7 ), while only $32 \%$ of mononucleated cells are differentiated into osteoclast cells after 10 days of osteoclast differentiation (Figure 8).

\section{Conclusions}

Mononucleated cells were shown to differentiate into osteoblast and osteoclast cells in their respective differentiation media, as shown by molecular biology, cell activity and morphology analyses. Osteoblast and osteoclast cells originated from different lineages, i.e., osteoblasts from mesenchymal stem cells and osteoclasts from haematopoietic stem cells. The capability of these mononucleated cells to generate different differentiated cells types indicates the plasticity of these adult stem cells. In conclusion, the ability of suspension mononucleated cells isolated from adult mouse peripheral blood to differentiate into both cells lineages demonstrates that these types of cells can be categorised as multipotent stem cells.

\section{Methods}

The animal experimental research has followed the animal guidelines provided by Faculty of Science and Technology, Universiti Kebangsaan Malaysia, which is similar to international animal guidelines.

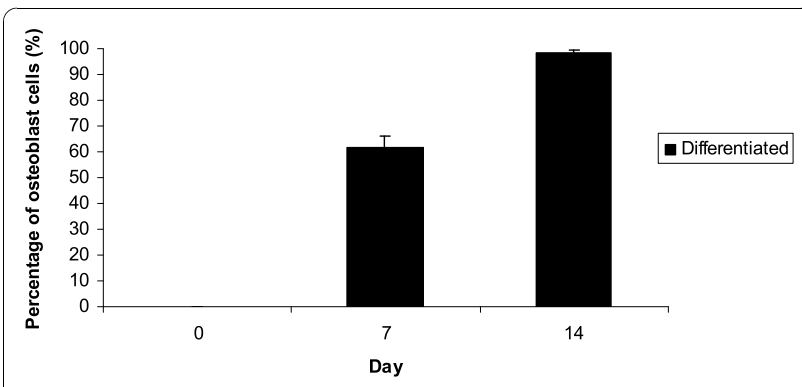

Figure 7 Morphology analysis of osteoblast differentiation. Von Kossa staining showed that $62 \%$ and $98 \%$ of mononucleated cells have been differentiated into osteoblast cells after cultured in osteoblast differentiation medium for 7 and 14 days, respectively. 


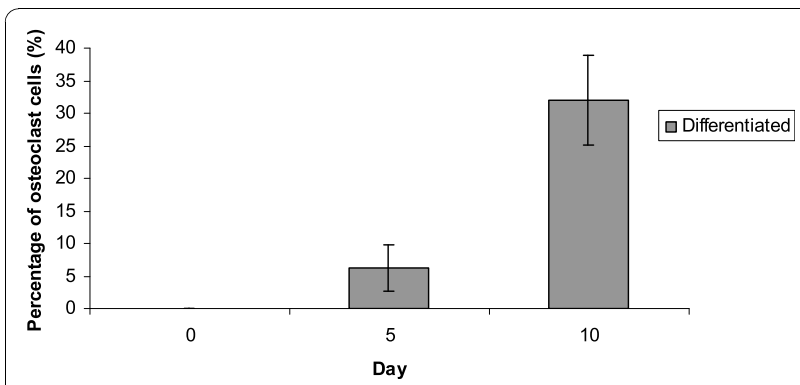

Figure 8 Morphology analysis of osteoclast differentiation. May-Grunwald-Giemsa staining showed that $6 \%$ and $32 \%$ of mononucleated cells have been differentiated into multinucleated osteoclast cells after cultured in osteoblast differentiation medium for 5 and 10 days, respectively.

\section{Isolation and Proliferation of Mononucleated Cells}

Mononucleated cells were isolated from mice by density gradient centrifugation using Ficoll-Paque ${ }^{\mathrm{TM}}$ Plus. The isolated cells were cultured in proliferation medium containing Alpha Minimal Essential Medium (AMEM) with $10 \%$ heat-inactivated Newborn Calf Serum (NBCS) and $2 \%$ penicillin/streptomycin. The cultures were incubated at $37^{\circ} \mathrm{C}$ in a fully humidified atmosphere containing $5 \%$ $\mathrm{CO}_{2}$. Each experiment was isolated from one mouse. Therefore for three independent experiments generated from our data were isolated from three different mice.

\section{Differentiation of Mononucleated Cells}

Suspension mononucleated cells were seeded at $1.0 \times$ $10^{5}$ cells $/ \mathrm{mL}$ in 24 -well plates for osteoblast and osteoclast differentiation assays. Cells cultured in complete medium were then supplemented with $50 \mu \mathrm{g} / \mathrm{mL}$ ascorbic acid and $10 \mathrm{mM} \beta$-glycerophosphate to induce differentiation into osteoblasts, while $50 \mathrm{ng} / \mathrm{mL}$ RANKL and $25 \mathrm{ng} / \mathrm{mL} \mathrm{M}$-CSF were added to induce osteoclast differentiation. The cultures were maintained at $37^{\circ} \mathrm{C}$ in a fully humidified atmosphere containing $5 \% \mathrm{CO}_{2}$. For control, the same cells were cultured with complete medium without supplementation of growth factors. Mononucleated cells viability in both differentiation and control mediums were assessed using trypan blue.

Molecular Analyses of Differentiated Mononucleated Cells Peripheral blood mononucleated cells have been isolated from three different mice and RT-PCR was done for each of them. Total RNA was isolated from approximately $1 \times 10^{5}$ cells that produce approximately $1.6 \mathrm{mg} /$ $\mathrm{mL}$ of RNA using TRI-Reagent (Sigma, USA) according to the manufacturer's instructions. Absorbance was measured at 260 and $280 \mathrm{~nm}$ to give ratio of A260:A280 was 1.8 to 2.0 in range to determine the samples purity. Total RNA was isolated from cells in differentiation medium and then reverse-transcribed using AMV reverse transcriptase and $\mathrm{T} f l \mathrm{DNA}$ polymerase according to the Access RT-PCR System protocol (Promega Corporation, USA). The primers sequences used were: 1) Gapdh, forward:

${ }^{5}$ CACTCCAATCGTCCCTACA ${ }^{3}$ and reverse: ${ }^{5}$ AAGG TGGAAGAGTGGGAG ${ }^{3}$, 2) Opn, forward: ${ }^{5}$ 'CACTCCAATCGTCCCTACA ${ }^{3}$ ' and reverse: ${ }^{5}$ GCTGCCCTT TCCGTTGTT ${ }^{3}$, and 3) Catk, forward: ${ }^{5}$ GGCAGGGTCCCAGACTCCAT ${ }^{3}$ ' and reverse: ${ }^{5}$ GTGTTGGTGGTGG GCTAC $^{3}$.

First strand cDNA synthesis was done at $45^{\circ} \mathrm{C}$ for 45 minutes. The pre-denaturation was done at $95^{\circ} \mathrm{C}$ for 2 minutes. The temperature cycle used to produce the second cDNA strand and RT-PCR amplification for Gapdh (denaturation: $94^{\circ} \mathrm{C}, 30 \mathrm{~s}$; annealing: $62^{\circ} \mathrm{C}, 60 \mathrm{~s}$; elongation: $68^{\circ} \mathrm{C}, 60 \mathrm{~s}$ ), $\mathrm{Opn}$ (denaturation: $68^{\circ} \mathrm{C}, 60 \mathrm{~s}$; annealing: $62^{\circ} \mathrm{C}, 60 \mathrm{~s}$; elongation: $68^{\circ} \mathrm{C}, 60 \mathrm{~s}$ ) and Catk (denaturation: $95^{\circ} \mathrm{C}, 30 \mathrm{~s}$; annealing: $63^{\circ} \mathrm{C}, 60 \mathrm{~s}$; elongation: $68^{\circ} \mathrm{C}, 60 \mathrm{~s}$ ) genes. After 40 cycles, elongation of the final strand was done at $68^{\circ} \mathrm{C}$ for 7 minutes. The RTPCR products were then analysed via electrophoresis of $1 \%$ agarose gels at $85 \mathrm{~V}$ using a 100-bp DNA marker (Vivantis). Each RT-PCR product was then subjected for sequencing and analysed.

\section{Osteoblastic and Osteoclastic Cell Analyses}

Cell activities for mononucleated cells cultured in osteoblast and osteoclast differentiation medium were determined using an osteologic disc. An osteologic disc is a disc coated with a micro layer of calcium phosphate on its surface. The cultured discs were stained using von Kossa staining on specific days, i.e., days 5, 7, 10 and 14 for osteoblast differentiation medium and days 5, 7 and 10 for osteoclast differentiation medium. The cell activities on the discs were observed using an inverted microscope (Olympus, Model: CKX75). Images were collected using a digital camera.

\section{Morphology Analyses of Differentiated Osteoblast and Osteoclast Cells}

Cytospin was used to analyze osteoblast and osteoclast morphology in this study. Approximately, 5-10 $\times 10^{5}$ cells/mL mononucleated cells were centrifuged at $78 \times$ $\mathrm{g}$ for 5 minutes. The pellet was smeared onto a glass slide and left to air-dry for about 1 to 2 hours. The cells were then stained with von Kossa and May-Grunwald-Giemsa stains to identify osteoblast and osteoclast cells, respectively. The slides were observed using an inverted microscope (Olympus, Model: CKX75). The percentages of differentiated cells were calculated based on a total of 200 cells counted randomly for approximately 5 different fields under microscope for each experiment. 


\section{List of abbreviations}

(ALP): Alkaline Phosphatase; (AMEM): Alpha Minimal Essential Medium; (Catk): Cathepsin K; (Col1): Collagen type I; (Gapdh): Glyceraldehyde-3-phosphate dehydrogenase; (M-CSF): Macrophage Colony-Stimulating Factor; (NBCS): Newborn Calf Serum; (Opn): Osteopontin; (PBS): Phosphate Buffer Saline; (RANKL): Receptor Activator NF- $\kappa$ B Ligand; (RT-PCR): Reverse TranscriptasePolymerase Chain Reactions; (TRAP): Tartrate Resistant Acid Phosphatase.

\section{Acknowledgements}

The authors would like to thank the Universiti Kebangsaan Malaysia for the financial grants (UKM-GUP-BTK-07-15-197 and UKM-OUP-KPB-33-170/2010) during this study.

\section{Author details}

${ }^{1}$ School of Biosciences and Biotechnology, Faculty of Science and Technology, Universiti Kebangsaan Malaysia, 43600 Bangi, Selangor, Malaysia. ${ }^{2}$ Department of Orthodontic, Faculty of Dentistry, Universiti Kebangsaan Malaysia, Jalan Raja Muda Abdul Aziz, 50300 Kuala Lumpur, Malaysia.

\section{Authors' contributions}

SHZA and RMAW developed the concept, designed and supervised the experiments. IZZA performed and developed experimental study and doing the analysis as part of PhD Thesis by research. MDY carried out part of the molecular and morphology analyses. All experiments and analysis were performed by IZZA and MDY, and approved by SHZA and RMAW. All authors read and approved the final manuscript before publication.

\section{Competing interests}

The authors declare that they have no competing interests.

Received: 7 July 2010 Accepted: 23 October 2010

Published: 23 October 2010

\section{References}

1. Shahrul Hisham ZA, Rohaya MAW, Ismanizan I, Nor Muhammad M, Zaidah ZA: Stem Cells, Cytokines and Their Receptors. APJMBB 2005, 13(1):1-13.

2. Shanthly N, Aruva MR, Zhang K, Mathew B, Thakur ML: Stem cells: a regenerative pharmaceutical. Q I Nucl Med Mol Imaging 2006, 50(3):205-216.

3. Shahrul Hisham ZA, Rohaya MAW, Intan Zarina ZA, Sahidan S, Nor Muhammad M, Zaidah Z: Stem Cell in Blood Development. Sains Malaysiana 2005, 34(1):21-26.

4. Yamane $\mathrm{T}$, Okuyama $\mathrm{H}$, Tsuneto $\mathrm{M}$, Hemmi H, Yamazaki $\mathrm{H}$, Hayashi Sl: Osteoclast lineage. In Handbook of Stem Cells. Edited by: Lanza R, Gearhart J, Hogan B, Melton D, Pedersen R, Thomson J, West M. London, Elsevier Academic Press; 2004:295-303.

5. Karsdal MA, Fjording MS, Foged NT, Delaissé JM, Lochter A: Transforming Growth Factor-beta-induced Osteoblast Elongation Regulates Osteoclastic Bone Resorption through a p38 Mitogen-activated Protein Kinase-and Matrix Metalloproteinase-dependent Pathway. J Biol Chem 2001, 276(42):39350-39358.

6. Miyamoto T, Suda T: Differentiation and function of osteoclasts. Keio J Med 2003, 52(1):1-7.

7. Filyushina ZG: Intravascular life span of granulocytes in thyrotoxicosis and diabetis mellitus. Bull Exp Biol Med 1969, 68(10):48-49.

8. Whitelaw DM: The Intravascular Lifespan of Monocytes. Blood 1966, 28(3):455-464

9. Sørensen AL, Rumjantseva V, Nayeb-Hashemi S, Clausen H, Hartwig JH, Wandall HH, Hoffmeister KM: Role of sialic acid for platelet life span: exposure of $\beta$-galactose results in the rapid clearance of platelets from the circulation by asialoglycoprotein receptor-expressing liver macrophages and hepatocytes. Blood 2009, 114(8):1645-1654.

10. Intan Zarina ZA, Shahrul Hisham ZA, Zaidah ZA, Rohaya MAW: Potential Differentiation of Three Types of Primitive Cells Originated from Different Proliferation Terms of Mouse Blood. Sains Malaysiana 2010, 39(2):305-313.

11. Swaminathan R: Biochemical markers of bone turnover. Clin Chim Acto 2001, 313(1-2):95-105.

12. Kartsogiannis V, Ng KW: Cell lines and primary cell cultures in the study of bone cell biology. Mol Cell Endocrinol 2004, 228(1-2):79-102.
13. Sodek J, Ganss B, McKee MD: Osteopontin. Crit Rev Oral Biol Med 2000, 11(3):279-303.

14. Wang D, Christensen K, Chawla K, Xiao G, Krebsbach PH, Franceschi RT: Isolation and Characterization of MC3T3-E1 Preosteoblast Subclones with distinct In Vitro and In Vivo Differentiation/Mineralization Potential. J Bone Miner Res 1999, 14(6):893-903.

15. Ziolkowskaa A, Rucinski M, Pucher A, Tortorella C, Nussdorfer GG, Malendowicz LK: Expression of osteoblast marker genes in rat calvarial osteoblast-like cells, and effects of the endocrine disrupters diphenylolpropane, benzophenone-3, resveratrol and silymarin. Chem Biol Interact 2006, 164(3):147-156.

16. Zhao Y, Guan H, Liu SF, Wu RC, Wang Z: Overexpression of QM induces cell differentiation and mineralization in MC3T3-E1. Biol Pharm Bull 2005, 28(8):1371-1376

17. Barber RD, Harmer DW, Coleman RA, Clark BJ: GAPDH as a housekeeping gene: analysis of GAPDH mRNA expression in a panel of human tissues. Physiol Genomics 2005, 21(3):389-395.

18. Hie M, Shimono M, Fujii K, Tsukamoto I: Increased cathepsin K and tartrate-resistant acid phosphatase expression in bone of streptozotocininduced diabetic rats. Bone 2007, 41(6):1045-1050.

19. Pang M, Martinez AF, Fernandez I, Balkan W, Troen BR: AP-1 stimulates the cathepsin K promoter in RAW 264.7 cells. Gene 2007, 403(1-2):151-158.

20. Faccio R, Takeshita S, Zallone A, Ross FP, Teitelbaum SL: c-FMS and the $a_{v} \beta_{3}$ integrin collaborate during osteoclast differentiation. J Clin Invest 2003, 111(5):749-758

21. Perez-Amodio S, Vogels IMC, Schoenmaker T, Jansen DC, Alatalo SL, Halleen JM, Beertsen W, Everts V: Endogenous expression and endocytosis of tartrate-resistant acid phosphatase (TRACP) by osteoblast-like cells Bone 2005, 36(6):1065-1077.

22. Roodman GD: Cell biology of the osteoclast. Exp Hematol 1999, 27(8):1229-1241

23. Woo KM, Kim H-M, Ko JS: Macrophage colony-stimulating factor promotes the survival of osteoclast precursors by up-regulating Bcl- $\mathrm{X}_{\mathrm{L}}$. Exp Mol Med 2002, 34(5):340-346.

24. Sindrey DR, Kusljie D, Kwong PC: Human parathyroid hormone (1-84) stimulates bone formation in rate bone marrow cultures during spaceflight. ASBMR 21st Annual Meeting 1999, SA424.

25. Cerovic A, Miletic I, Sobajic S, Blagojevic D, Radusinovic M, El-Sohemy A: Effects of Zinc on the Mineralization of Bone Nodules from Human Osteoblast-like Cells. Biol Trace Elem Res 2007, 116(1):61-71.

26. Voronov I, Heersche JNM, Casper RF, Tenenbaum HC, Manolson MF: Inhibition of osteoclast differentiation by polycyclic aryl hydrocarbons is dependent on cell density and RANKL concentration. Biochem Pharmacol 2005, 70(2):300-307.

27. Valverde $\mathrm{P}, \mathrm{Tu} \mathrm{Q}, \mathrm{Chen} \mathrm{J}$ : BSP and RANKL induce osteoclastogenesis and bone resorption synergistically. J Bone Miner Res 2005, 20:1669-1679.

28. Valverde P, Zhang J, Fix A, Zhu J, Ma W, Tu Q, Chen J: Overexpression of Bone Sialoprotein Leads to an Uncoupling of Bone Formation and Bone Resorption in Mice. J Bone Miner Res 2008, 23(11):1775-1788.

29. Alhadlaq A, Mao JJ: Tissue-engineered Neogenesis of Human-shaped Mandibular Condyle from Rat Mesenchymal Stem Cells. J Dent Res 2003, 82(12):951-956.

30. Tsuang YH, Sun JS, Chen LT, Sun SCK, Chen SC: Direct effects of caffeine on osteoblastic cells metabolism: the possible causal effect of caffeine on the formation of osteoporosis. J Orthop Surg Res 2006, 1:7.

31. Yagi M, Miyamoto T, Sawatani Y, Iwamoto K, Hosogane N, Fujita N, Morita K, Ninomiya K, Suzuki T, Miyamoto K, Oike Y, Takeya M, Toyama Y, Suda T: DC-STAMP is essential for cell-cell fusion in osteoclasts and foreign body giant cells. J Exp Med 2005, 202(3):345-351.

32. Li Z, Kong K, Qi W: Osteoclast and its roles in calcium metabolism and bone development and remodeling. Biochem Biophys Res Commun 2006, 343(2):345-350.

33. Servet-Delprat C, Arnaud S, Jurdic P, Nataf S, Grasset MF, Soulas C, Domenget C, Destaing O, Rivollier A, Perret M, Dumontel C, Hanau D, Gilmore GL, Belin MF, Rabourdin-Combe C, Mouchiroud G: Flt3+ macrophage precursors commit sequentially to osteoclasts, dendritic cells and microglia. BMC Immunol 2002, 3:15.

doi:10.1186/1478-811X-8-29

Cite this article as: Ariffin et al: Differentiation analyses of adult suspension mononucleated peripheral blood cells of Mus musculus. Cell Communication and Signaling 2010 8:29. 